\title{
Morphological - Iesional aspects of prosthetic mitral valve thrombosis
}

\section{- Forensic implications -}

"University "Ovidius", Faculty of Medicine, Department of Forensic Pathology, Constanţa, Romania

2 "Mina Minovici" National Institute of Legal Medicine, Bucharest, Romania

${ }^{3}$ Forensic County Service, Constanţa, Romania

\begin{abstract}
The purpose of this paper is to present a case of prosthetic mitral valve thrombosis in a young patient with a history of acute articular rheumatism with bivalvular damage which required prosthetic mitral twin disc and tricuspid annuloplasty that, despite effective anticoagulation treatment, shows a high degree of mitral valve obstruction with severe hemodynamic disturbances that ultimately led to death.

The particularity of the case lies in the development of thrombosis in twin disc prosthesis type, complication that, in the literature, is cited as being more rarely met than the cases of single-disc prosthesis.
\end{abstract}

Keywords: prosthesis, mitral valve, thrombosis, anticoagulant therapy, acute articular rheumatism

\section{Popa M. F.}

Associate Professor, MD, PhD, Department of Forensic Pathology, Faculty of Medicine, University "Ovidius" Constanţa Forensic County Service, 2 Zmeurei Alley, Constanța, Romania Tel. +40744297697

mariusppopa2005@yahoo.com

\section{Introduction}

Mechanical valve dysfunction has the most important cause- prosthetic thrombosis. This can be massive-obstructive (in this case) or incomplete unobtrusive

Obstructive thrombosis of the prosthesis is a severe complication potentially fatal, occurred after valvular replacement that requires urgent diagnosis and treatment, which is defined by the presence of any thrombus, in the absence of infection, attached or located in close proximity to prosthesis, which partially obstructs the flow of blood through prosthesis or interferes with its function $[1,15]$.

The incidence of thromboembolic complications in prosthesis is between $0.03 \%$ and $4.3 \%$ patient / year.[3] Prosthetic thrombosis occurs in the mechanical prosthesis and especially when positioned in place of the mitral valve affected.

The main reason for the occurrence of this complication, is the presence of a coagulation defect with an index of anticoagulation monitoring (INR) often below the target, but which can however intervene (in most cases) and other factors 
among which the most common being : heart stasis attractiveness of the left atrial wall whose ultrasound examination appears hypertrophied size of $45 \mathrm{~mm}$ and thrombus formation in the left atrial cavity, which often creates the appearance of background heart rhythm disturbances in atrial fibrillation.

Prosthetic valve thrombosis is more common in patients with coagulation defect shortly after surgery, in the presence of risk factors susceptible to thrombosis (type of the prosthesis and the position in which is assembled, pathologies and / or associated diseases such as: the presence of LV dysfunction and atrial fibrillation; hyper coagulant status, pregnancy and neoplastic pathologies).

A valve selection is made according to patient's age, valvular pathology and its echo on the patient's quality of life, availability of the type of valve and last but not least, according to the index risk / benefit in favor of the patient, in this case, mechanical twin disc prosthesis for a young patient with high life expectancy, is being recommended.

\section{Materials and methods}

The case selected is part of casuistry of Constanta County Forensic Services in 2013.

As provided in Article $185 \mathrm{CP}$ "forensic autopsy is mandatory in cases of violent death or when death is suspicious" (death "occurred shortly after or simultaneously with medical intervention, surgical diagnosis or treatment") if the presented case fulfills the conditions within the forensic case.

The purpose of this paper is to present, identify and analyze the morphological - lesional substrate which led to the death of a patient with acute articular rheumatism with known cardiac disease, who received prosthetic mitral valve with twin disc prosthesis and tricuspid annuloplasty with Carpentier-Edwards ring and who, despite a anticoagulant therapy dosed accurately and efficiently, valvular thrombosis developed a complication that could not be prevented by the specific treatment given.

\section{Case presentation}

Forensic autopsy was performed based on a service order presented before Constanta County Forensic Service by the research bodies, which, in order to solve the file on the person in question, have established the following objectives:

a. Nature and medical cause of death

b. If there are signs of violence on the body, layout, features and the mechanism of their production c. If between injury and death there is no causal link

d. If lesions have vital and post vital features.

\section{Medical history of disease}

From the medical documents submitted, it appears that male sex person aged 43 years, is known with medical history of acute articular rheumatism with bivalvular damage (mitral and tricuspid valve), for which in 2011, he had a surgery of replacing the damaged mitral valve with a metal prosthesis and is performed a tricuspid annuloplasty. Anticoagulant therapy followed by it, is per bone-with Sintrom.

The patient presents himself in the ER, complaining of palpitations and dry cough, symptoms which started in the evening two weeks before presentation to the hospital. Imaging tests performed at admission (trans-thoracic echocardiography / transabdominal) have detected the following changes: mobilizing this disc prosthesis; AS, VS dilated; intense spontaneous contrast, no thrombus in VS; filling and emptying low speeds; dysfunctional mitral twin disc prosthesis, lateral hemi-disc locked in closed position attached thrombus in both hemi-discs (maximum thickness $7 \mathrm{~mm}$ in the lateral hemi-discs); average gradient transprosthetic level $11 \mathrm{mmHg}$, mitral ring $31 \mathrm{~mm}$

Thus, the patient is being hospitalized in the cardiology department with the diagnosis of mitral mechanical prosthesis with twin discs - observation prosthesis dysfunction, thrombosis of prosthesis. Second degree of aortic insufficiency. First degree of tricuspid insufficiency. Tricuspid annuloplasty with Carpentier-Edwards ring. 
During the 7 days of hospitalization, the patient receives treatment with heparin, under constant monitoring of the aPTT value.

The oral-maxillary-facial surgery examination performed (in terms of underlying pathology) reveals no focal lesions, while imaging examination (radiological) lung, highlights slightly enlarged hilum and deepened peribronchial vascular, bilateral.

The ECG exam detects: sinus rhythm, left apical hypertrophy and TA of $12 / 8 \mathrm{mmHg}$.

In the last days of hospitalization, the patient presents mucopurulent expectoration cough, without pulmonary auscultator modifications, which after two days of onset associates dyspnea, expressed in $\mathrm{SaO} 2$ of $90 \%$ and a hemodynamic imbalance with oTA of $77 / 40 \mathrm{mmHg}$ and $\mathrm{AV}$ of $88 \mathrm{~b} / \mathrm{min}$

The patient experiences symptoms suggestive of acute pulmonary edema, supported also by radiological examination performed, at which point, IOT with VM and cardiovascular emergency surgery is being decided. In the operating room, the patient develops cardiac arrest by electromechanical dissociation, unresponsive to resuscitation heart made through both internal and external cardiac massage, declaring him exitus.

\section{Necropsy examination:}

External examination:

When performing external examination of the body, there have been found numerous excoriated placards arranged above the chest, abdomen, anterior right side thigh through its entire length, consisting of multiple escorial stripes of maximum size 4 / 0.3, arranged anarchic, covered by a fine, brown, sticky crust.

In addition, there was evidence of medical and surgical treatment, consisting of: median incision edges presternal affront by sutures of $8 / 0.2 \mathrm{~cm}$; left forearm 1/3 distal and anterior, vertical incision of $2 /$ $0.2 \mathrm{~cm}$ suture, vein punctures on the dorsal hand and fold of the right elbow.

\section{Internal examination:}

Cord of $14 / 10 / 5 \mathrm{~cm}$ in size, empty heart cavities, enlarged volume, valvular device with tricuspid annuloplasty with Carpentier-Edwards ring and metal mitral prosthesis covered entirely by thrombus with disappeared mobility. Myocardium with numerous spots, streaks and areas with a diameter of $0.5 \mathrm{~cm}$, whitish, indurated; VS thickness of about $1.5 \mathrm{~cm}$.

General macroscopic examination reveals:

Signs of medical and surgical treatment, generalized visceral stasis, incipient atheromatosis, cerebral edema, bilateral hydrothorax, acute pulmonary edema, cardiomegaly eccentric, tricuspid annuloplasty with Carpenter-Edwards ring, mitral metal prosthesis thrombosis, myocardial sclerosis, previously infiltrated mediastinal bleeding, liver, heart, old splenectomy, thyroid hypertrophy.

\section{Forensic conclusions}

Death of the undersigned was not violent, being produced by cardio-circulatory failure, consequence of acute mitral metal prosthetic thrombosis in a person with acute articular rheumatism with impaired mitral and tricuspid bivalvular and multiple preexisting pathologies and / or associated basic diseases (atheromatosis and incipient cerebral, systemic and coronary atherosclerosis, chronic liver stasis, cardiomegaly, myocardial fibrosis, left side eccentric ventricular hypertrophy).

Primary traumatic lesions (excoriations) found at necropsy, were able to produce shortly before death by striking tangential hard body and are not the causal link with the medical cause of death.

\section{Discussions}

Although valvular diseases of rheumatic origin have decreased as incidence in developed countries, they continue to be the cause of significant morbidity and mortality worldwide [2,3]. In developing countries, cases of mitral stenosis in adolescents and adults are meet, where the prophylaxis of acute articular rheumatism has been practiced in optimal conditions for decades in such countries [4].

The goal of surgery is to restore the competence of the valve, either by valve replacement or by valve repairing through different surgical techniques. 
A valve replacement is performed when the valve is altered, and which is not properly fulfilling its role when it trains alarming symptoms such as respiratory discomfort, dyspnea and which evolves until rest or when it can give rise to serious complications represented by acute pulmonary edema $[13,16,20]$; this is indicated especially in congenital valvular diseases or acquired due to certain endocarditic, malformations, tumours, articular rheumatism, etc.

Acute articular rheumatism (AAR) is a systemic inflammatory disease of the connective tissue caused by an abnormal immune response to an anterior pharyngo-tonsillar infection of group A beta haemolytic streptococcus [18]. This, in a prone body, causes a humoral and cellular immune response for advocating a number of factors such as latency period of about 3 weeks between streptococcal infection and the development and demonstration of crossreactivity between AAR streptococcus antigens and human proteins

Cases of rheumatic carditis prevalence reach their peak at 30-40 years [7]. Therefore, although acute articular rheumatism is a disease that begins in childhood, adults, particularly young adults during maximal activity, feel its effects. It essentially affects the heart, joints and central nervous system manifesting as a febrile illness, apparently selflimiting, but with cardiac squeals, mainly valvular, as shown in this case.

Frequently interested in the rheumatic attack are the heart valves, which are flammable, making rheumatismale endocarditis. With healing of the rheumatic attack, the inflammation of the valves also yields, which is followed by sclerosis, welding or retraction of the valve apparatus, leading to the narrowing (stenosis), either to the complete lack of tightness of closing valves (failure) $[18,19]$. Usually, rheumatic heart damage occurs in the first rheumatic spurt.

There is a linear relationship between the severity of mitral regurgitation produced in the first episode and subsequent chronic rheumatic heart damage frequency $(90 \%$ of cases with severe mitral regurgitation which develops chronic valvular diseases). It is estimated that worldwide there are 15.6 million people with rheumatismale heart diseases, annually registering 470,000 new cases and 233,000 deaths (Figure 1).

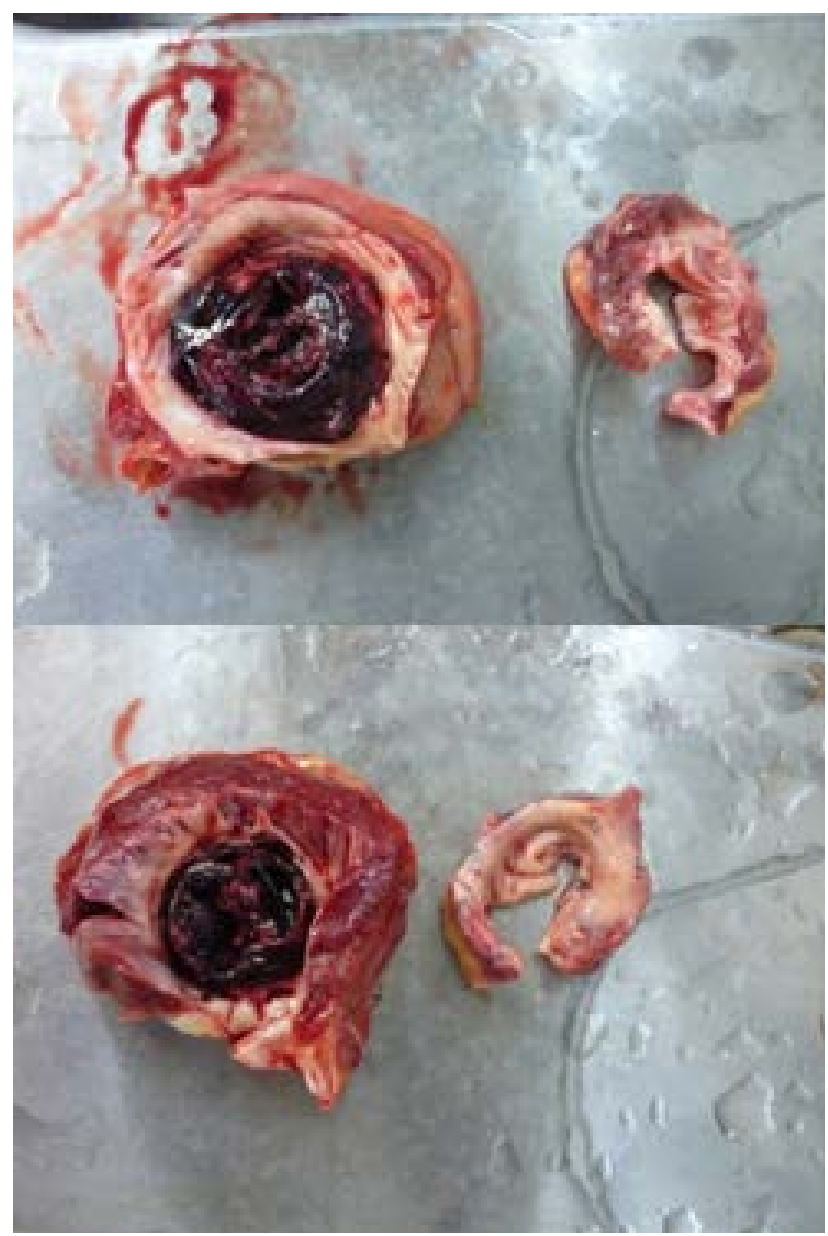

Figure 1 - Massive mitral prosthetic valve thrombosis and tricuspid annuloplasty piece.

Treatment is indicated as with any valve disease, firstly by medication, so that in case this is exceeded by the course of the disease, to resort to surgical treatment by fitting a prosthetic valve, as was done in our case $[9,10]$.

In terms of therapeutic strategies in prosthetic valve thrombosis, this issue remains a controversial topic.

Treatment of prosthetic valve thrombosis, looked into briefly in the ACC / AHA of 1997 guidebook, may be medically performed by thrombolysis or surgically, but without firm recommendations.

1. Thrombectomy or valvular prosthetic 
replacement represents the treatment of choice; however, postoperative mortality is high, ranging between $8 \%$ and $20 \%$; operated for emergency cases, mortality is $37-54 \%$ and even higher for those in critical condition [25].

2. Thrombolysis has emerged as a very interesting therapeutic alternative since 1980, in patients where surgical risk was considered increased; the method of thrombolysis is still controversial because of the risk of fragmentation of the thrombus and systemic embolism occurrence of irreversible consequences; however, many authors propose thrombolysis as a treatment of choice prosthetic valve thrombosis, its results being encouraging.

Thrombolysis is recommended for massive obstructive thrombosis. Roudaut et al have reported an increased efficiency of thrombolytic treatment to the aortic prosthesis $(85 \%)$ compared to the mitral $(63 \%)$ [23], in a considerable number of patients.

Thrombolysis is contraindicated in the event of nonobstructive thrombosis and of a stroke recently documented by $\mathrm{CT}$ or immediately after surgery; otherwise if a recent stroke, surgery is also contraindicated.

The disadvantages of thrombolytic treatment are represented by the possibility of systemic embolization or of thrombosis recurrence, the latter explanation being the persistence of "pannus" $[4,5,6]$.

In the case presented, where we are dealing with a young patient without severe damage of multiple organs, timing of surgery was tried, initially opting for the anticoagulant treatment, whose doses were adjusted according to the value of APTT's assessed twice daily, following that during the evolution of the existing pathology, the decision for performing surgery to be taken.

At the necropsy examination of the valve apparatus, the normal functioning of the mechanical twin disc prosthesis has been checked, while, the necropsy technique appropriate steps have been taken in advance, as follows: opening the left atrium and washing the agonizing clot (postmortem format), cross sectioning of the aorta in the supravalvular ridge and the middle portion of the left ventricle in short axis. Thus, they could inspect both sides of the mechanical valve prosthesis. The agonizing clot (postmortem) is cruoric (red), smooth, elastic, adherent, glossy, wet, unlike the thrombus (ante mortem) which is adherent, rough, brittle, tough (Figure 2).

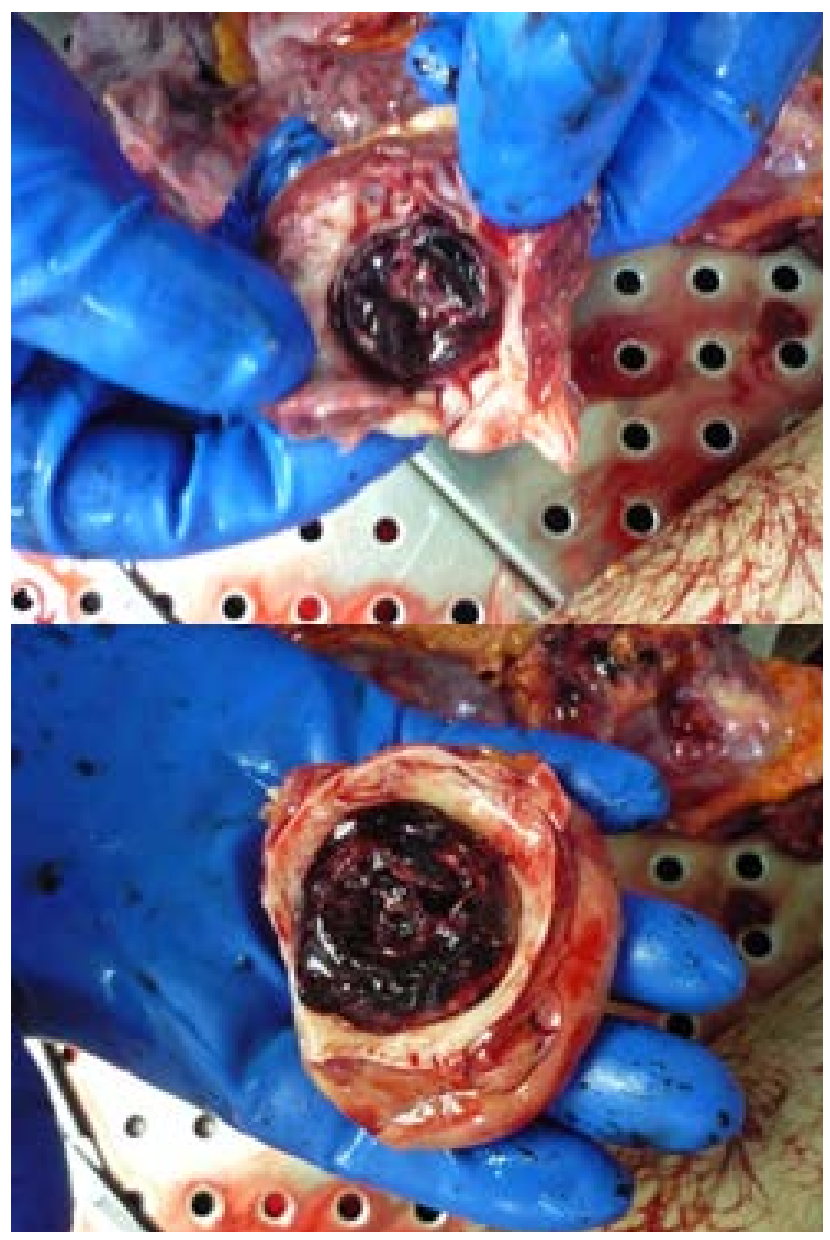

Figure 2 - Massive mitral prosthetic valve thrombosis, with strongly adherent thrombus, scarlet red color, dry, brittle that completely obstructs the opening hole of the prosthesis.

Mechanical valves positioned, with an evolution for at least several months, develop a tissue white fiber layer over the suture ring, which protrudes the ends of the suture. This layer is often called neointimal and it seems that its role is to reduce thrombus formation. The projection of this layer can be produced like a pannus over the valvular orifice with blood flow obstruction. 


\section{Conclusions}

In the evolution of acute articular rheumatism, when damage also occurs at heart valves, mechanical prosthesis of valves is commonly performed, these having a prolonged resistance but with the disadvantage of a lifetime anticoagulation therapy. However, although anticoagulant treatment is well dosed under INR control [17], (as in this case), the presence of risk factors is an individual claim which cannot be ignored and unfortunately incomplete prevented, and in this case has contributed to the occurrence of thrombosis to which was also added the background of a low therapeutic compliant, prosthetic valve thrombosis being in this case, massive - obstructive, with negative consequences.

Forensic aspects of the case presented aims at assessing the real possibilities of complete diagnosis and thus at anticipating complications and recommendations appropriate to evolutionary process termination interventional surgical thrombectomy / replacement valve thrombosis, by establishing the rate of each factor in the causal of death, including medical care.

In other words, we consider that the patient has received adequate medical-surgical treatment, correctly prescribed and performed on time, based on compliance tests with good professional practice in health care both during valve prosthesis and also when evolutionary complication occurred, the more in the case of the unforeseeable circumstances presented.

\section{References}

1. Vahanian, A., Lung, B., Pierard, L., Dion, R. \& Pepper, L. (2009). Prosthetic valve surgery In Oxford University Press (Eds.), Camrn, A.J., Lucher, T., Serruys, P. The ESC textbook of cardiovascular medicine, second ed (pp. 806-
817)

2. Jacob, R. \& Troughton, R. (2009). Prosthetic heart valve. In Wolters Kluwer/Lippincott (Eds.), Griffin, B., Topol, E. Manual of cardiovascular medicine, third ed. (pp. 251-265)

3. Otto, C. \& Bonow, R. (2008). Prosthetic cardiac valve. In Saunders Elsevier (Eds.), Libby, P., Bonow, R., Mann, D., Zipes, D. Braunwald's Heart Disease, eighth ed. (pp. 1682-1693)

4. Acar, C. \& Theodore, P. (2010). Surgery for valvular heart disease. In Mosby-Elsevier (Eds.), Crawford, M., DiMarco, J., Paulus, W. Cardiology, third ed. (pp. 1345-1378)

5. Rahitmoola, S., Wu, Y.X., Grunkemeier, G. \& Starr, A. (2008). Prosthetic heart valve: choice of valve and management of patient In Mc GrawHill Medical (Eds.), Fuster, V., O'Rourke, R., Walsh, R., PoolWilson, P. Hurst's The Heart, twelfth ed. (pp. 1783-1799)

6. Garcia, M. (2007). Prosthetic valve. In Lippincott Williams Wilkins (Eds.), Topol, E. Textbook of cardiovascular medicine, third ed. (pp. 389-401)

7. Vahanian, A., Baumgartner, H. \& Bax, J. et al. (2001). Guidelines on the management of valvular heart disease. Eur Heart J., 28, 230-268

8. Bonow, R.O., Carabello, B.A. \& Chateljee, K. et al. (2006). ACC/AHA guidelines for the management of patients with valvular heart disease. J Am Coll Cardiol, 48(3), e1-148

9. Nishimura, R.A., Carabello, B.A. \& Faxon, D.P. et al. (2008). ACC/AHA guidelines update on valvular heart disease. $J$ Am Coll Cardiol, 52, 676-685

10. Zoghbi, W., Chambers, J. \& Dumensnil, J. el al. (2009). Recommendations for evaluation of prosthetic valves with echocardiography and Doppler ultrasound. A report from ASE's guidelines and standards comrnitee and the task force on prosthetic valves. ASE, 22(9), 975-1014

11. Vongpatanasin, W., Hillis, D. \& Lange, R. (1996). Prosthetic heart valves. $N$ Eng J Med, 335(6), 407-416

12. Pibarot, P. \& Dusmeshil, J.G. (2009). Prosthetic heart valves Selection of optimal prosthesis and long-term management. Circulation, 119, 10341048

13. Botzenhardt, F., Eichinger, W. \& Bleiziffer, 
S. el al. (2005). Haemodynamic comparison of bioprostheses for complete supra annuIar position in patients with small aortic annulus. $J$ Am Coll Cardiol, 45, 2054-2060

14. Webb, J. (2008). Percutaneous aortic valve replacement will become a common treatment for aortic valve disease. $\mathrm{J} \mathrm{Am}$ Coll Cardiol, 1, 122-126

15. Vahanian, A. \& Otto, C. (2010). Risk stratification of patients with aortic stenosis. Eur Heart $J$, DOI: 10.1093/Eurheart 1/ehp 575

16. Scherptong, R., Vliegen, H. \& Winter, M. el al. (2009). Tricuspid valve surgery in adults with a dysfunctional systemic right ventricle. Repair or replace? Circulation, 119, 1467-1472

17. Lurz, P., Coats, L. \& Khambadkone, S. el al. (2008). Percutaneous pulmonary valve implantation. Impact of evolving technology and leaning curve on clinical outcome. Circulation, 117, 1964-1972

18. Montorsi, P., Cavoretto, D. \& Alimento, M. et al. (2003). Prosthetic mitral valve thrombosis: can fluoroscopy predict the efficacy of thrombolytic treatment? Circulation, 08 (suppl. II) II: 79-84

19. Pflederer, T. \& Flachskampf, F. (2010). Echocardiographic follow-up after heart valve replacement. Heart, 96, 75-85
20. Schievano, S., Migliavacca, F. \& Coats, L. el al. (2007). Percutaneous pulmonary valve implantation based on rapid prototypion on right ventricular outflow tract and pulmonary trunk from MR data. Radiology, 242(2), 490-497

21. Barbetseas, J., Nagueh, S. \& Pitsavos, C. el al. (1998). Differentiating thrombus from pannus formation in obstructed mechanical prosthetic valves: an evaluation of clinical, transthoracic and transesophageal echocardiographic parameters. J Am Coll Cardiol, 32, 1410-1417

22. David, T. (2005). Is prosthesis-patient mismatching a clinically relevant entity? Circulation, 111, 3186-3187

23. Hoffinan, G., Lutter, G. \& Cremer, L. (2008). Durability of bioprosthetic cardiac valves. Dtsch Artzeb, 106(8), 143-148

24. Colii, A., Verhoye, J.P. \& Leguerrier, A. el al. (2007). Anticoagulation and antiplatelet therapy of bioprosthetic heart valve recipients: an unresolved issue. Eur J Cardio-thoracic Surgery, $31,573-577$

25. Elkayan, U. (2005). ValvuIar heart disease and pregnancy. J Am Coll Cardiol, 46, 403-410 